\title{
MMW-HOTLINE
}

Leser der MMW können sich mit allen Fragen zur Abrechnung und Praxisführung an Helmut Walbert, Facharzt für Allgemeinmedizin, Würzburg, wenden. Sie erreichen ihn jeden Donnerstag von 13 bis $15 \mathrm{Uhr}$ unter der kostenlosen Rufnummer (0800) 2379830 oder per E-Mail: w@lbert.info.

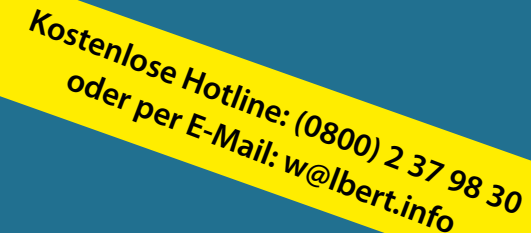

Helmut Walbert

Allgemeinarzt, Medizinjournalist und Betriebswirt Medizin
Widerspruch in den Krebsfrüherkennungsrichtlinien

\section{Kann das sein: Koloskopie ab 55 - Beratung erst später?}

\author{
Dr. med. P. S., KV Berlin \\ Im Rahmen des Darmkrebsmonats versu- \\ chen wir unsere Patienten gezielt anzu- \\ sprechen. Ist es richtig, dass ein Patient ab \\ dem 55. Lebensjahr einen Anspruch auf \\ die Vorsorgekoloskopie hat, aber erst nach \\ Vollendung des 55. Lebensjahres zu dieser \\ Untersuchung beraten werden kann? \\ Antwort: Richtig! Sie haben einen Wider- \\ spruch zwischen den Gebührenordnungs- \\ positionen (GOP) im EBM und den Krebs- \\ früherkennungsrichtlinien gefunden. Ich
}

habe bereits wiederholt darauf aufmerksam gemacht. Leider hat sich bis heute an diesem Textfehler nichts geändert.

In den Krebsfrüherkennungsrichtlinien des G-BA im § 37 „Anspruch auf Maßnahmen zur Früherkennung von kolorektalen Karzinomen" steht im Absatz (3) „Versicherte haben ab dem Alter von 55 Jahren Anspruch auf insgesamt zwei Koloskopien zur Früherkennung des kolorektalen Karzinoms". Im § 38, „Beratung über Maßnahmen zur Früherkennung von kolorektalen Karzinomen" steht:
(1) „Die Ärztin oder der Arzt hat die oder den Versicherten möglichst frühzeitig ab dem Alter von 50 Jahren einmalig über das Gesamtprogramm eingehend zu informieren" und bei (2) "Möglichst bald ab dem Alter von 55 Jahren sollen die Versicherten eine weitere Beratung (zweite Beratung) erhalten".

Die dazu gehörige GOP 01 740, „Beratung zur Früherkennung des kolorektalen Karzinoms" enthält allerdings in der Beschreibung des obligaten Leistungsinhaltes die Bestimmung: „Möglichst frühzeitig nach Vollendung des 55. Lebensjahres". Damit ist die erste Beratung nicht abrechenbar und die zweite Beratung erst mit Beginn des 56. Lebensjahres. Verstehe das, wer kann, oder aber die Beratung ist offensichtlich nur für die Uneinsichtigen oder Beratungsresistenten abrechenbar.

\section{Bestimmung von Blutzucker und Quickwert}

\section{Wer darf die Ergebnisse mitteilen?}

Frau Dr. med. S. T., Allgemeinärztin, KVSA Ich habe eine qualifizierte MFA. Darf diese Mitarbeiterin in meinem Auftrag Auskunft über Untersuchungsergebnisse wie beispielsweise Quick- und Zuckerwerte erteilen?

Antwort: Grundsätzlich ja, wenn die „Spielregeln" vorher genau festgelegt sind. Dennoch ist die Beratung erst einmal - und dazu zählt auch die Mitteilung erhobener Untersuchungsergebnisse eine persönliche ärztliche Leistung. Delegation ist immer dann möglich, wenn es sich um

Leistungen handelt, die in Schwierigkeit und Gefährlichkeit keine Gefährdung für den Patienten bedeuten. Diese Delegation ist sicherlich bei den von Ihnen angefragten

Blutzuckerwerten möglich, wenn die MFA entsprechend geschult ist und bei der Beratung die notwendigen Konsequenzen beurteilen kann.

Darf eine Arzthelferin Laborergebnisse mitteilen?
Dies gilt erst recht für die Interpretation erhobener INR-Werte. Hier verordnen wir ja bisweilen Geräte und Teststreifen zur QuickSelbstkontrolle durch den Patienten, wenn der Patient nach entsprechender Schulung, dazu befähigt wurde. Warum sollte dies also durch eine geschulte MFA nicht möglich sein?

Ich empfehle Ihnen allerdings, mindestens einmal pro Jahr Ihre Mitarbeiterin zu überprüfen, indem Sie bei einigen Beratungsgesprächen dabei sind. Diese sollten mit Namen des Patienten und Datum dokumentiert werden. Auf diese Weise können Sie Ihre Verantwortung und die Wahrnehmung der Aufsichtspflicht nachweisen. 